\title{
The Agrin/MuSK Signaling Pathway Is Spatially Segregated from the Neuregulin/ErbB Receptor Signaling Pathway at the Neuromuscular Junction
}

\author{
Jonathan C. Trinidad, ${ }^{1}$ Gerald D. Fischbach, ${ }^{2}$ and Jonathan B. Cohen ${ }^{1}$ \\ ${ }^{1}$ Department of Neurobiology, Harvard Medical School, Boston, Massachusetts 02115, and 2National Institute of \\ Neurological Disorders and Stroke, Bethesda, Maryland 20892
}

\begin{abstract}
The neuregulin/erbB receptor and agrin/MuSK pathways are critical for communication between the nerve, muscle, and Schwann cell that establishes the precise topological arrangement at the vertebrate neuromuscular junction (NMJ). ErbB2, erbB3, and erbB4 as well as neuregulin, agrin, and MuSK are known to be concentrated at the NMJ. Here we have examined NMJs from gastrocnemius muscle of adult rat using immunofluorescence confocal microscopy to characterize in detail the distribution of these proteins relative to the distribution of acetylcholine receptors (AChRs). We have determined that erbB2 and erbB4 are enriched in the depths of the secondary junctional folds on the postsynaptic muscle membrane. In contrast, erbB3 at the NMJ was concentrated at presynaptic terminal Schwann cells. This distribution strongly argues that erbB2/erbB4 het-
\end{abstract}

At the mammalian neuromuscular junction (NMJ), the motor neuron axon terminal lies in a trough on the muscle surface known as the primary gutter. There, Schwann cell processes cap axon terminals. Within the primary gutter, the muscle surface gives rise to additional invaginations of $\sim 1 \mu \mathrm{m}$ in depth (secondary folds). The primary gutter (including the crests intervening between the secondary folds) and the secondary folds represent distinct subdomains of the NMJ. Many proteins are enriched at the NMJ within a particular subdomain (for review, see Sanes and Lichtman, 1999). For example, acetylcholine receptor (AChR) and rapsyn (a protein associated with the AChR and required for its localization) are enriched in the primary gutter, whereas voltage-gated sodium channels are enriched in secondary folds (Flucher and Daniels, 1989). Dystrophin, a membrane-associated cytoskeletal protein required for membrane stability, is enriched in secondary folds, whereas the dystrophin homolog utrophin is enriched in the primary gutter (Bewick et al., 1992, 1996).

Acetylcholine receptor-inducing activity (ARIA) and glial growth factor II (GGF II) are products of the neuregulin-1 gene, which is subject to extensive alternative splicing (for review, see Fischbach and Rosen, 1997). GGF II, which is synthesized as a soluble, secreted factor, promotes proliferation and survival of Schwann cells (Marchionni et al., 1993). ARIA is synthesized by motor neurons as a transmembrane precursor that is cleaved to release the extracellular ligand. ARIA binds to erbB receptor tyrosine kinases on muscle (Goodearl et al., 1995), initiating a signal transduction cascade (Altiok et al., 1997; Fromm and Burden, 1998; Si et al., 1999) that regulates AChR mRNA transcription

Received July 20, 2000; revised Sept. 15, 2000; accepted Sept. 21, 2000.

This work was supported in part by United States Public Health Service Grant NS18458 and a Stuart H. P. and Victoria Quan Fellowship in Neurobiology (J.C.T.) We thank Professor Elio Raviola for the electron micrograph and many helpful suggestions concerning immunofluorescence microscopy. We also thank Dr. Andrew Goodearl for the anti-neuregulin antiserum HM 24.

Correspondence should be addressed to Jonathan B. Cohen, Department of Neurobiology, Harvard Medical School, 220 Longwood Avenue, Boston, MA 02115. E-mail: jonathan_cohen@hms.harvard.edu.

Copyright (C) 2000 Society for Neuroscience $0270-6474 / 00 / 208762-09 \$ 15.00 / 0$ erodimers are the functional postsynaptic neuregulin receptors of the NMJ. Neuregulin was localized to the axon terminal, secondary folds, and terminal Schwann cells, where it was in a position to signal through erbB receptors. MuSK was concentrated in the postsynaptic primary gutter region where it was codistributed with AChRs. Agrin was present at the axon terminal and in the basal lamina associated with the primary gutter region, but not in the secondary junctional folds. The differential distributions of the neuregulin and agrin signaling pathways argue against neuregulin and erbB receptors being localized to the NMJ via direct interactions with either agrin or MuSK.

Key words: neuregulin; erbB receptor; agrin; MuSK; neuromuscular junction; immunohistochemistry

by muscle nuclei (Martinou et al., 1991; Chu et al., 1995; Sapru et al., 1998).

ErbB2 and erbB3 are enriched at the NMJ, although reports regarding erbB4 are inconsistent (Altiok et al., 1995; Moscoso et al., 1995; Zhu et al., 1995; Lin et al., 2000). At the NMJ, it is unknown which combination of erbB heterodimers or homodimers is formed. However, not all possible dimer combinations are functional (Guy et al., 1994; Tzahar et al., 1994; Chen et al., 1996; Pinkas-Kramarski et al., 1996). Additionally, individual dimer combinations differ in their signal potency (Lenferink et al., 1998; Waterman et al., 1998).

Agrin is a heparan sulfate proteoglycan released from axon terminals (Ferns et al., 1992; Hoch et al., 1993) where it binds to the MuSK receptor tyrosine kinase complex on muscle, leading to clustering of AChRs (for review, see Glass and Yancopoulos, 1997). Mutant mice homozygous for a disruption in either the agrin or MuSK genes have severely disrupted NMJs (Dechiara et al., 1996; Gautam et al., 1996). Ectopic expression of neural agrin or constitutively active MuSK in noninnervated regions of muscle results in aggregation of NMJ-specific proteins, including erbB receptors (Cohen et al., 1997; Meier et al., 1998; Jones et al., 1999).

Biochemical and heterologous expression experiments support a model where activated MuSK forms a scaffold around which rapsyn self-associates and recruits AChRs. Rapsyn expressed in nonmuscle cells is targeted to the plasma membrane where it is organized into aggregates. Coexpressed AChR is recruited to these aggregates (Froehner et al., 1990; Phillips et al., 1991; Ramarao and Cohen, 1998). MuSK and rapsyn expressed in non-muscle cells colocalize, and in rapsyn-deficient mice, AChRs are diffusely distributed, whereas MuSK remains localized in the postsynaptic membrane (Apel et al., 1997).

ErbB3 is not enriched at NMJs of rapsyn-deficient mice, whereas the presence or absence of erbB2 and erbB4 has not been examined (Gautam et al., 1995). This observation, and the results of ectopic agrin/MuSK expression, led us to ask what were the relative locations of components of the neuregulin and agrin signaling pathways. Here we demonstrate that at NMJs of adult rat gastroc- 
nemius muscle, components of these two pathways are spatially segregated, with neuregulin, erbB2, and erbB4 enriched in the depths of the secondary folds, whereas agrin and MuSK are enriched in the primary gutter.

\section{MATERIALS AND METHODS}

Muscle tissue. Adult Sprague Dawley rats were anesthetized with $10 \mathrm{mg} / \mathrm{kg}$ Nembutal (Abbott Laboratories) and perfused through the heart with 150 $\mathrm{ml}$ PBS, pH 7.1 and then with $250 \mathrm{ml}$ of a $2 \%$ formaldehyde/PBS solution (from paraformaldehyde). The gastrocnemius muscle was dissected, and the muscle was placed in a $20 \%$ sucrose/PBS solution at $4^{\circ} \mathrm{C}$ until the tissue sank. Muscle blocks were embedded in OCT (Sakura), frozen, and stored at $-80^{\circ} \mathrm{C}$ until needed. To obtain cross sections of muscle, the blocks of tissue were cut perpendicular to the axis of the muscle fibers. To obtain en face and side view images, longitudinal sections of muscle were cut parallel to the axis of the muscle fibers.

Antibodies. The rabbit anti S-100 polyclonal antibody (Z 311) was obtained from Dako (Glostrup, Denmark) and used at a 1:1000 dilution. Mouse monoclonal antibodies against utrophin (DRP1) and dystrophin (DYS1 and DYS2) were obtained from NovoCastra Laboratories (Newcastle upon Tyne, UK), and all were used at a 1:10 dilution of hybridoma supernatants. Hybridoma supernatant of a mouse antibody to SV2 was a gift from Kathy Buckley (Harvard Medical School) and was used at a 1:25 dilution. Rabbit polyclonal antibodies against erbB2 (SC-284) and erbB3 (SC-285) were from Santa Cruz Biotechnology (Santa Cruz, CA); each was used at a 1:200 dilution. Staining was markedly reduced by coapplication of the respective blocking peptides (SC-284 P and SC-285 P). The mouse monoclonal anti-erbB3 (05-390) and rabbit polyclonal anti-erbB4 (06-572) antibodies were from Upstate Biotechnology (Lake Placid, NY); each was used at a 1:100 dilution. A pan-agrin mouse monoclonal antibody (AGR550 ) was from StressGen (Victoria, British Columbia, Canada) and used at a 1:200 dilution. Rabbit polyclonal antibodies to MuSK (anti-MuSK/Nsk2 and anti-Cyt-MuSK) were gifts from Markus Ruegg (University of Basel) and Werner Hoch (Max Plank Institut für Entwicklungsbiologie), respectively. They were used at a 1:50 and 1:10 dilution and gave similar results Anti-neuregulin antibody 1914 was produced by immunizing rabbits with human met-NDF- $\alpha_{14-241}$, and it was used at a 1:50 dilution (Sandrock et al., 1995). Anti-neuregulin antiserum HM 24 was produced by immunizing rabbits with a peptide specific for the GGF II isoform of neuregulin, the 11 amino acid $\beta 3$ exon (STSTPRLSLPE), coupled to keyhole limpet hemocyanin. It was used at a 1:100 dilution. Staining was markedly reduced by coapplication of the peptide used as the immunogen. Bodipy-FLconjugated $\alpha$-bungarotoxin (Bodipy- $\alpha \mathrm{Btx}$ ), Cy3-conjugated goat antirabbit, and $\mathrm{Cy} 3$-conjugated goat anti-mouse antibodies were obtained from Molecular Probes (Eugene, OR). They were all used at a 1:500 dilution.

Immunohistochemistry. Muscle sections were cut at a thickness of $10 \mu \mathrm{m}$ in a cryostat (Microm HM 500 OM) and used immediately. The sections were placed onto Superfrost/Plus microscope slides (Fisher Scientific, Pittsburgh, PA). The samples were air-dried, washed for 5 min with PBS, then blocked for $30 \mathrm{~min}$ in a PBS solution containing $10 \%$ normal goat serum (NGS), $2 \%$ fish gelatin, and $10 \mathrm{mg} / \mathrm{ml}$ bovine serum albumin (BSA) Primary antibody was diluted in PBS containing 10\% NGS and $10 \mathrm{mg} / \mathrm{ml}$ $\mathrm{BSA}$, then applied to the sections overnight at $4^{\circ} \mathrm{C}$. The sections were washed three times for $5 \mathrm{~min}$ with PBS. The appropriate secondary antibody was then applied along with the Bodipy- $\alpha$ Btx in a solution of PBS containing $10 \% \mathrm{NGS}, 0.2 \%$ fish gelatin, and $10 \mathrm{mg} / \mathrm{ml} \mathrm{BSA}$ for $1 \mathrm{hr}$ at room temperature. The sections were washed three times for $5 \mathrm{~min}$. A $5 \%$ solution of $N$-propylgallate in $90 \%$ glycerol was added to the sections, and coverslips were applied and sealed with nail polish.

Light microscopy. Epifluorescence images were obtained using a Nikon Eclipse E800 equipped with a Nikon $20 \times$ Plan Apo objective $[0.75$ numerical aperture (NA)]. Bodipy-FL fluorescence was filtered through a Nikon 91617 FITC, HYQ bandpass filter (emission 515-560 nm). Cy3 fluorescence was filtered through a Nikon 96176 Rhodamine, HYQ bandpass filter (emission 590-650 $\mathrm{nm}$ ). No bleed-through of fluorescent signal was observed when only one fluorophore was used. Images were acquired with a Micromax digital camera (Princeton Instruments) operated using Metamorph software (Universal Imaging Corporation). Confocal microscopy was performed using a Bio-Rad MRC-1024 laser-scanning unit coupled to a Nikon Eclipse E600 with a Nikon $60 \times$ Plan Apo objective (1.40 NA). All confocal images were acquired using a constant iris setting to preserve the thickness of the focal plane. Laser intensity and gain were adjusted on a per image basis to optimally use the full linear range of the camera. The Bodipy- $\alpha$ Btx and the Cy3-conjugated secondary antibody were excited sequentially using the 488 and $568 \mathrm{~nm}$ excitation lines of the krypton/argon mixed gas laser. No bleed-through of signals across channels was observed. For en face images, the $z$-axis was adjusted for each NMJ to find the focal plane for which the Bodipy- $\alpha \mathrm{Btx}$ and $\mathrm{Cy} 3$-conjugated secondary antibody images were both in focus. When the Cy3-conjugated secondary antibody was localized presynaptically, a focal plane between the presynaptic staining and the primary gutter was chosen. At this focal plane, the Bodipy$\alpha \mathrm{Btx}$ staining preferentially highlighted the upper edges of the primary gutter, with less intense staining at the bottom of the primary gutter. All digital images were processed using Adobe PhotoShop. The contrast was not adjusted on any of the confocal images.

Electron microscopy. Anesthetized rats were perfused through the heart with $2 \%$ formaldehyde and $2.5 \%$ glutaraldehyde in Sörensen phosphate buffer, $\mathrm{pH} 7.4$, after the vascular tree was rinsed with physiological solution. Fragments of gastrocnemius muscle were then kept in the same fixative for $2 \mathrm{hr}$ at room temperature. They were subsequently post-fixed in osmium ferrocyanide and stained en bloc first with uranyl acetate and subsequently with tannic acid. After they were embedded in EponAraldite, thin sections were stained with uranyl and lead, and micrographs were obtained with a Jeol 1200EX electron microscope.

\section{RESULTS}

\section{Localization of proteins at the NMJ}

Initially, we examined cross sections of adult rat gastrocnemius muscle to confirm that the antibodies we were using revealed the expected enrichment of neuregulin, erbBs, agrin, and MuSK at the NMJ (Fig. 1). NMJs were identified by labeling with Bodipy- $\alpha$ Btx. Consistent with previous results, erbB2, erbB3, and erbB4 were enriched at the NMJ. There was no clear evidence for extrajunctional sarcolemmal staining with any of the antibodies. Immunoreactivity for erbB2 and erbB4 (but not erbB3) was also found at the myotendinous junction (data not shown). Antiserum (HM 24) against the $\beta 3$ isoform of neuregulin as well as an antibody (1914) against the entire extracellular domain of an Ig-containing isoform $(\alpha 2)$ both stained the NMJ but not the extra-junctional sarcolemma. The immunofluorescence signal from two MuSK antibodies showed enrichment at the NMJ, with no staining evident in extra-junctional sarcolemma. An antibody recognizing all agrin isoforms showed strong immunofluorescence at the NMJ. There was no detectable signal from the extra-junctional muscle cell basal lamina, although there was slight staining of non-muscle cells.

In addition, we examined the distributions of antigens serving as markers to identify subdomains of the NMJ. The antigens were the synaptic vesicle protein SV2 (Buckley and Kelly, 1985) and the Schwann cell calcium binding protein S-100 (Trachtenberg and Thompson, 1997), as well as utrophin and dystrophin, proteins enriched in the postsynaptic primary gutter and secondary folds, respectively (Bewick et al., 1992). All four of the marker proteins showed enrichment at the NMJ. SV2, S100, and utrophin were essentially confined to the NMJ, whereas dystrophin was also observed to stain the surrounding sarcolemma. At the level of resolution obtained using a $20 \times$ objective, there were slight differences in the distributions of SV2, S-100, and dystrophin relative to $\alpha$-Btx. However, the differences were not sufficient to readily distinguish the subdomains.

\section{Electron microscope analysis of the NMJ}

Consideration of the ultrastructure of a representative NMJ (Fig. $2 A$ ) provides a guide indicating how the different subdomains appear when analyzed by immunofluorescence confocal microscopy [see also Bewick et al. (1992)]. If the NMJ were viewed in cross section (Fig. 2B), both the axon terminal and terminal Schwann cells would appear offset above the primary gutter region. The secondary folds would appear offset below the primary gutter region. If the NMJ were rotated so that it was viewed en face, the axon terminal would appear largely confined to the primary gutter region. The Schwann cells would appear associated with but not completely confined to the primary gutter region, because (as seen in the cross section) the Schwann cell processes extend beyond it laterally. The secondary folds would have a broader distribution than the primary gutter because of the fact that they extend both downward and laterally into the cytoplasm.

\section{Confocal characterization of marker proteins}

Using confocal immunofluorescence microscopy on longitudinal sections of rat adult gastrocnemius muscle, we examined how the distribution of marker proteins for the NMJ subdomains compared with the distribution of $\mathrm{AChR}$, when the NMJ was viewed in en face and side view images (Fig. $2 C-N$ ). Muscle sections were cut longitudinally, parallel to the surface of the muscle fibers. Muscle cut in this manner will have NMJs on the surface of the sarcolemma distributed evenly about the $360^{\circ}$ axis of the muscle fiber. NMJs can be viewed en face when they are aligned parallel to the 

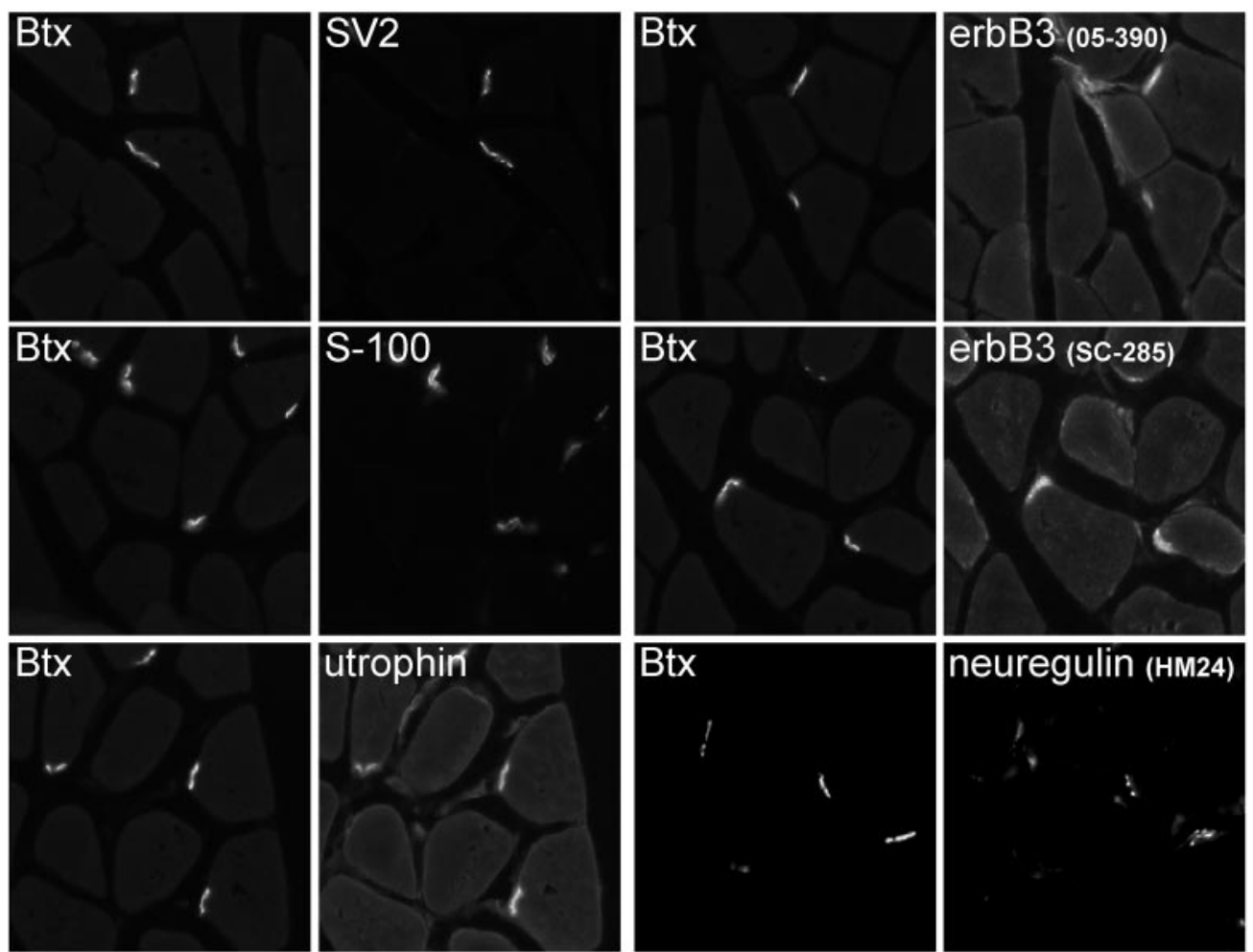

Figure 1. Immunofluorescence visualiza-
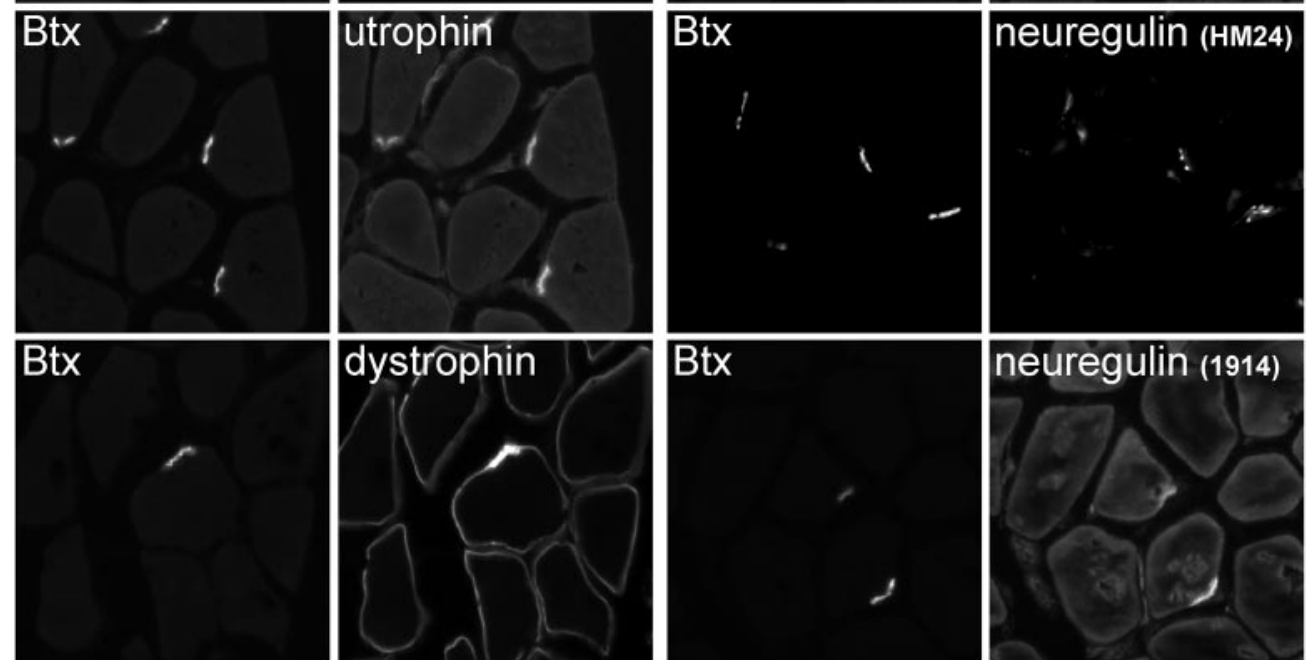

tion of proteins enriched at the NMJ. Pro-
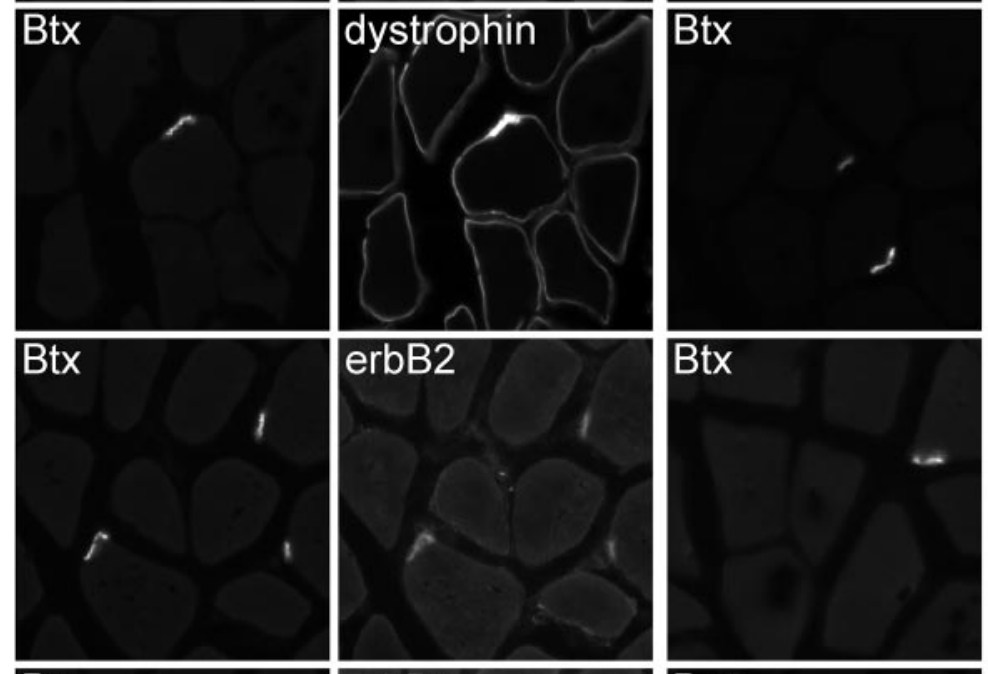

teins of the agrin/MuSK and neuregulin/ erbB signaling pathways as well as marker proteins for subdomains of the NMJ were visualized in cross sections of adult rat gastrocnemius muscle using a $20 \times$ objective. Bodipy- $\alpha$ Btx was used to identify individual NMJs. The axon terminal marker SV2, Schwann cell marker S-100, utrophin (a protein enriched in the postsynaptic primary gutter), and dystrophin (a protein enriched in the secondary folds) all show clear staining at the NMJ. Dystrophin additionally shows the expected enrichment along the sarcolemma. Immunofluorescence from antibodies against erbB2, erbB3, erbB4, neuregulin, agrin, and MuSK all show enrichment at the NMJ with little extra-junctional staining of the muscle. Scale bar, $200 \mu \mathrm{m}$.
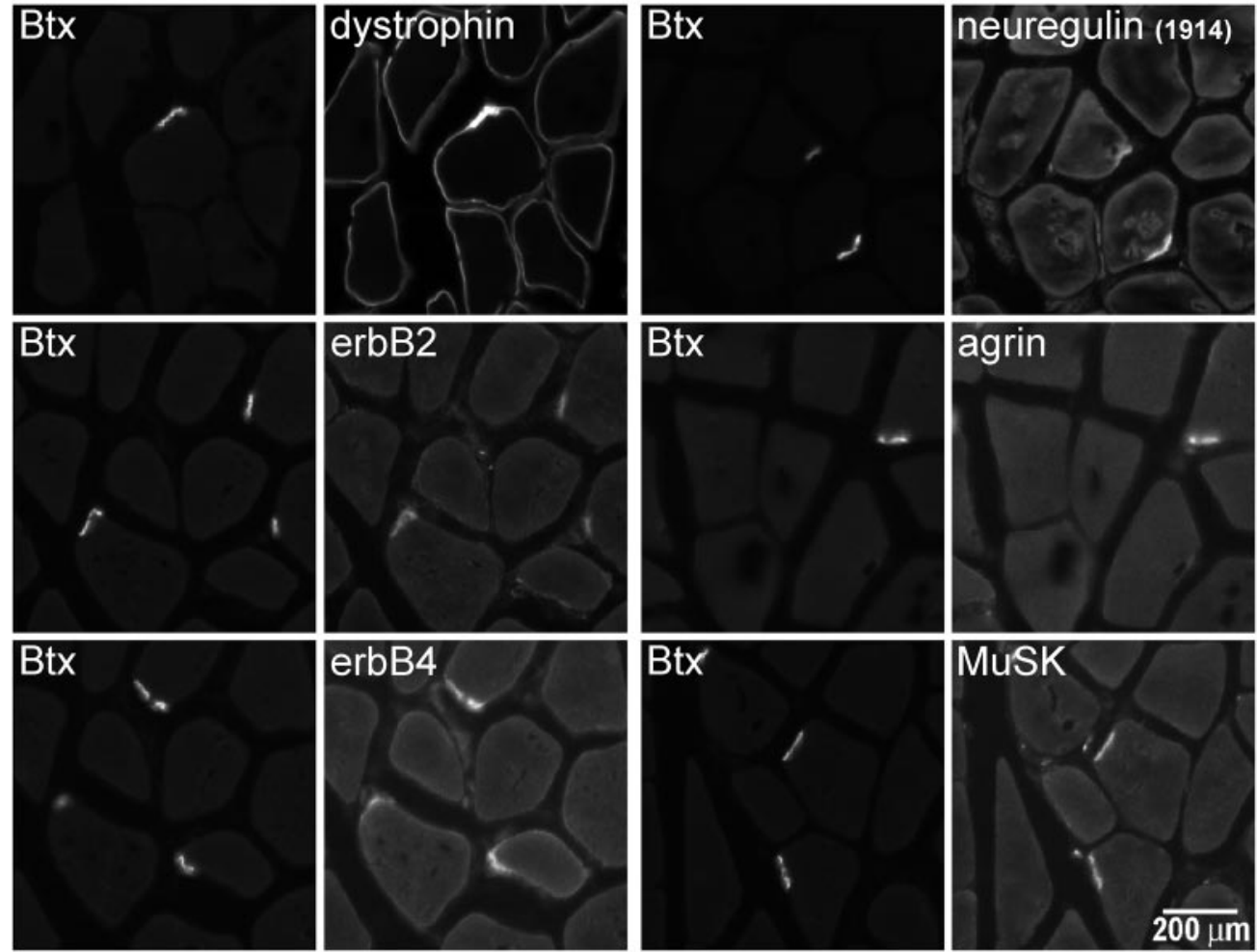

focal plane of observation. Side views were obtained when NMJs on the surface of the fiber were oriented perpendicular to the plane of focus. In a side view, the distribution of AChRs in the primary gutter region visualized using Bodipy- $\alpha$ Btx generally appeared as a line on the surface of the sarcolemma, often with scalloped depressions corresponding to individual primary gutters. The localization of subdomains in side view images should be essentially equivalent to their localization in cross-section images. The side view images in Figures 2-4 have been rotated such that the muscle fibers are oriented horizontally with the NMJs on the top surface of the sarcolemma and presynaptic structures above the primary gutter region.
When the distribution of the axon terminal marker SV2 was examined en face (Fig. 2C), the immunoreactivity appeared localized within the primary gutter region. Interestingly, the SV2 immunoreactivity was neither continuous nor completely coextensive with Bodipy- $\alpha$ Btx. Rather the staining was largely confined to the central portion of the primary gutter and appeared punctate in nature. When examined in side view (Fig. 2D), SV2 immunoreactivity was offset presynaptically, above the primary gutter (as defined by Bodipy- $\alpha$ Btx fluorescence). The punctate regions of SV2 fluorescence occurred within the "scallops" of the Bodipy- $\alpha$ Btx. Figure $2 E$ is a diagram representing the distribution of SV2 relative to Bodipy- $\alpha$ Btx. 


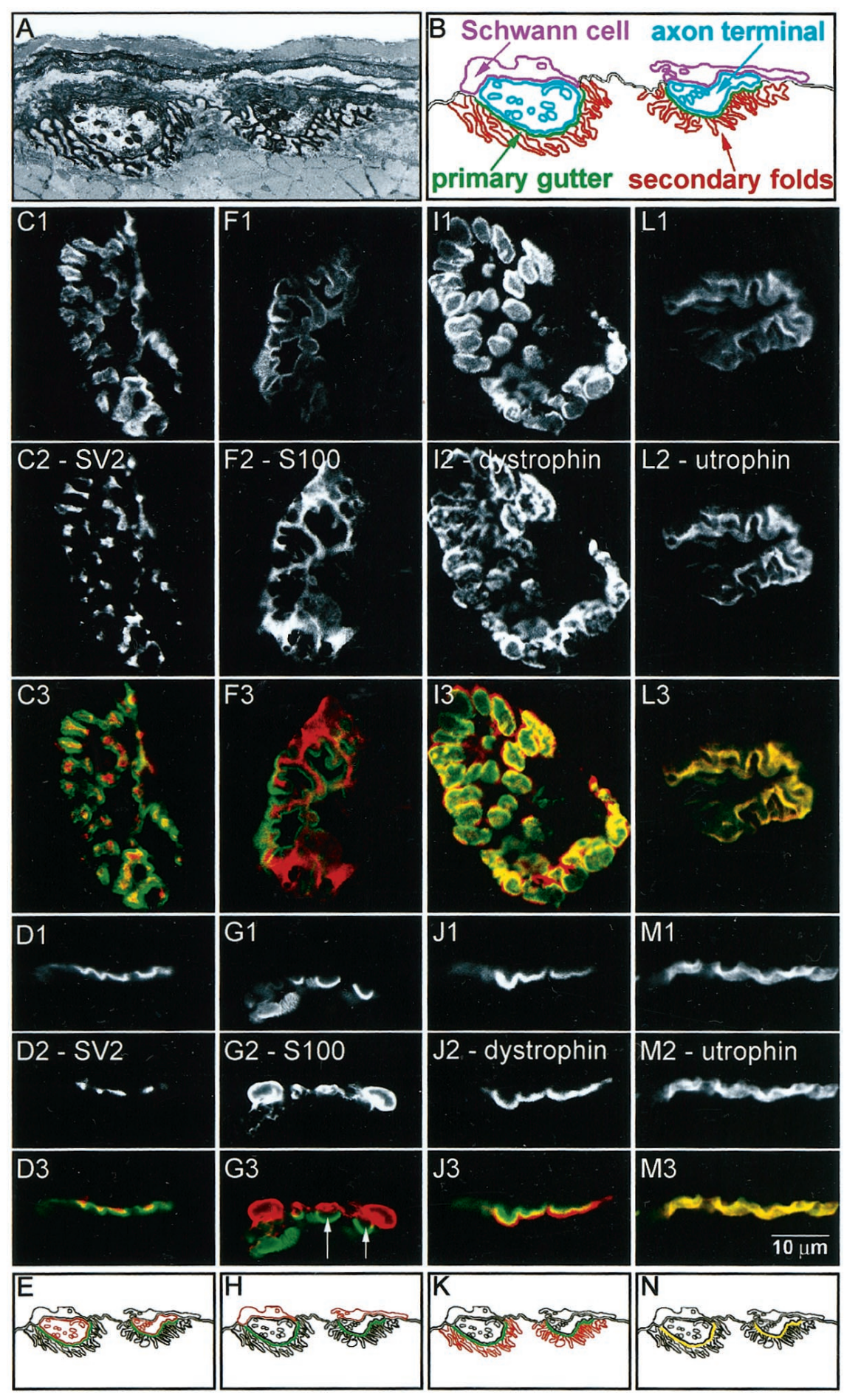

Figure 2. Confocal immunofluorescence analysis of marker proteins for the subdomains at adult rat gastrocnemius NMJ. $A$, Electron micrograph of the NMJ at $1800 \times$ magnification. The basal lamina was stained using tannic acid. In this image, two axon terminals lying in primary gutters are evident. The overlying Schwann cells and underlying secondary folds can also be seen. $B$, A schematic of the electron micrograph showing the relative orientations of the subdomains of the NMJ: Schwann cell (purple), axon terminal (cyan), primary gutter (green), and secondary folds (red). En face $(C 1, F 1, I 1, L 1)$ and side view $(D 1, G 1, J 1, M 1)$ images of NMJs stained with Bodipy- $\alpha$ Btx. The NMJs were double-labeled with antibodies against SV2 (C2, $D 2), \mathrm{S}-100(F 2, G 2)$, dystrophin $(I 2, J 2)$, and utrophin (L2, M2) and visualized using a Cy3-conjugated secondary antibody. Color overlays are shown with $\alpha$-Btx in green and the respective antibody in red (C3-M3). Schematics of these distributions are shown in $E, H, K$, and N. SV2 is localized to the axon terminal. S-100 is localized to the Schwann cell, and gaps between the primary gutter and the capping Schwann cell can often be seen (G3, arrows). Dystrophin is enriched in the secondary folds. Utrophin is coextensive with $\alpha$-Btx in the primary gutter. Scale bar, $10 \mu \mathrm{m}$.
When the distribution of S-100, the Schwann cell marker, was examined en face (Fig. $2 F$ ), the immunoreactivity was largely confined to the primary gutter region. In contrast to SV2, the S-100 staining was more continuous. This is consistent with a labeling of Schwann cell processes capping the nerve terminal. The staining could also be seen to deviate away from the primary gutter to potentially label the Schwann cell bodies. In side view (Fig. 2G), S-100 immunofluorescence was often clearly offset above the Bodipy- $\alpha$ Btx signal, and a gap (indicated by arrows in Fig. 2G3) could be seen between the S-100 immunofluorescence and the Bodipy- $\alpha$ Btx signal. This was consistent with the Schwann cell processes capping the axon terminal at the NMJ. Figure $2 \mathrm{H}$ is a diagram representing the distribution of $\mathrm{S}-100$ relative to Bodipy- $\alpha$ Btx.
Examined en face, the pattern of immunoreactivity for dystrophin, which is enriched in the secondary folds, was qualitatively similar to that of Bodipy- $\alpha$ Btx (Fig. 2I). However, when the two images are overlaid (Fig. 2I3), it can be seen that the dystrophin signal was more broadly distributed than Bodipy- $\alpha$ Btx, and the region of most intense dystrophin immunofluorescence was just outside the region of Bodipy- $\alpha$ Btx fluorescence (Bewick et al., 1996). The distribution of dystrophin therefore appeared to rim the primary gutter region. Side view images also showed the pattern of dystrophin fluorescence to be different from that of Bodipy- $\alpha$ Btx (Fig. 2J). An overlay of the two images revealed that dystrophin immunofluorescence was offset below the Bodipy- $\alpha$ Btx fluorescence, corresponding to dystrophin in the secondary folds. There was a partial overlap of the two fluorescence signals, perhaps 
corresponding to low levels of dystrophin in the primary gutter, but the most intense dystrophin signal was distinctly offset from the most intense Bodipy- $\alpha$ Btx region. Dystrophin immunofluorescence did not obviously extend fully into the region labeled by Bodipy- $\alpha$ Btx.

Utrophin, which is enriched in the primary gutter (Bewick et al., 1992), had an immunofluorescence distribution strikingly similar to that of Bodipy- $\alpha$ Btx in both en face and side view images (Fig. $2 L, M)$. Overlay of the images revealed an almost perfect overlap. The distributions of Bodipy- $\alpha$ Btx and utrophin were nearly identical.

\section{Distribution of erbB receptors at the NMJ}

The distributions of erbB receptors are shown in Figure 3. Antibodies against erbB2 (Fig. $3 A, B$ ) and erbB4 (Fig. $3 G, H$ ) have distributions consistent with both erbB2 and erbB4 localized to the secondary folds. En face images of erbB2 and erbB4 showed that the most intense regions of immunoreactivity occurred just outside the primary gutter region (Fig. $3 A, G$ ). When examined in side view, the signals from erbB2 and erbB4 were clearly seen offset below the primary gutter region (Fig. $3 B, H$ ).

The distribution of erbB3 was strikingly different and consistent with erbB3 localization to terminal Schwann cells. Similar results were obtained with both the monoclonal and polyclonal anti-erbB3 antibodies. In en face views (Fig. 3D), erbB3 immunoreactivity appeared mostly confined to the primary gutter. There was a continuous distribution of the immunofluorescence, which at parts extended outside the primary gutter region. When examined in side view (Fig. $3 E$ ), erbB3 was offset presynaptically from the primary gutter. A gap (indicated by arrow in Fig. 3E3) could be seen between the erbB3 staining and the primary gutter, similar to the gap seen with S-100. These staining patterns were consistent with erbB3 labeling in both Schwann cell bodies and Schwann cell processes.

\section{Distribution of neuregulin at the NMJ}

A neuregulin antibody (1914) generated against the extracellular domain of neuregulin ( $\alpha 2$ isoform) labeled both the nerve terminal and the secondary folds, albeit with a higher signal at the nerve terminal. When viewed en face (Fig. $4 A$ ), neuregulin immunoreactivity could be seen enriched in two subregions. The strongest immunofluorescence was localized to the primary gutter region, with weaker fluorescence observed outside the primary gutter region (consistent with the secondary folds). The staining in the primary gutter was not continuous, but rather was concentrated in punctate regions consistent with neuregulin associated with axon terminals. When examined in side view (Fig. 4B), neuregulin immunoreactivity was also detected in two distinct subregions. There was an intense presynaptic immunofluorescence localized just above the troughs of the primary gutter (Fig. 4B2, arrow). There was also less intense immunofluorescence, offset below the primary gutter, in the secondary folds (Fig. 4B2, arrowhead).

The en face and side view images of neuregulin immunoreactivity, as defined by the antiserum against the GGF II ( $\beta 3)$ isoform (HM 24), had a distribution consistent with the association of this neuregulin isoform with Schwann cells. In en face images (Fig. 4D), the immunoreactivity was not confined to the primary gutter. The staining pattern was very similar to S-100, and it appeared as if Schwann cell processes as well as Schwann cell bodies were labeled. In side view (Fig. $4 E$ ), the immunoreactivity was clearly offset presynaptically from the Bodipy- $\alpha$ Btx signal.

\section{Distribution of MuSK and agrin at the NMJ}

The distribution of MuSK immunoreactivity at the NMJ indicated that MuSK was mainly concentrated in the primary gutter region. When viewed en face (Fig. $4 G$ ), MuSK immunoreactivity was in a pattern very similar to Bodipy- $\alpha$ Btx, although there were low levels of MuSK immunofluorescence outside the primary gutter. When the images were overlaid, the regions of most intense MuSK signal overlapped with regions of most intense Bodipy- $\alpha$ Btx signal, which
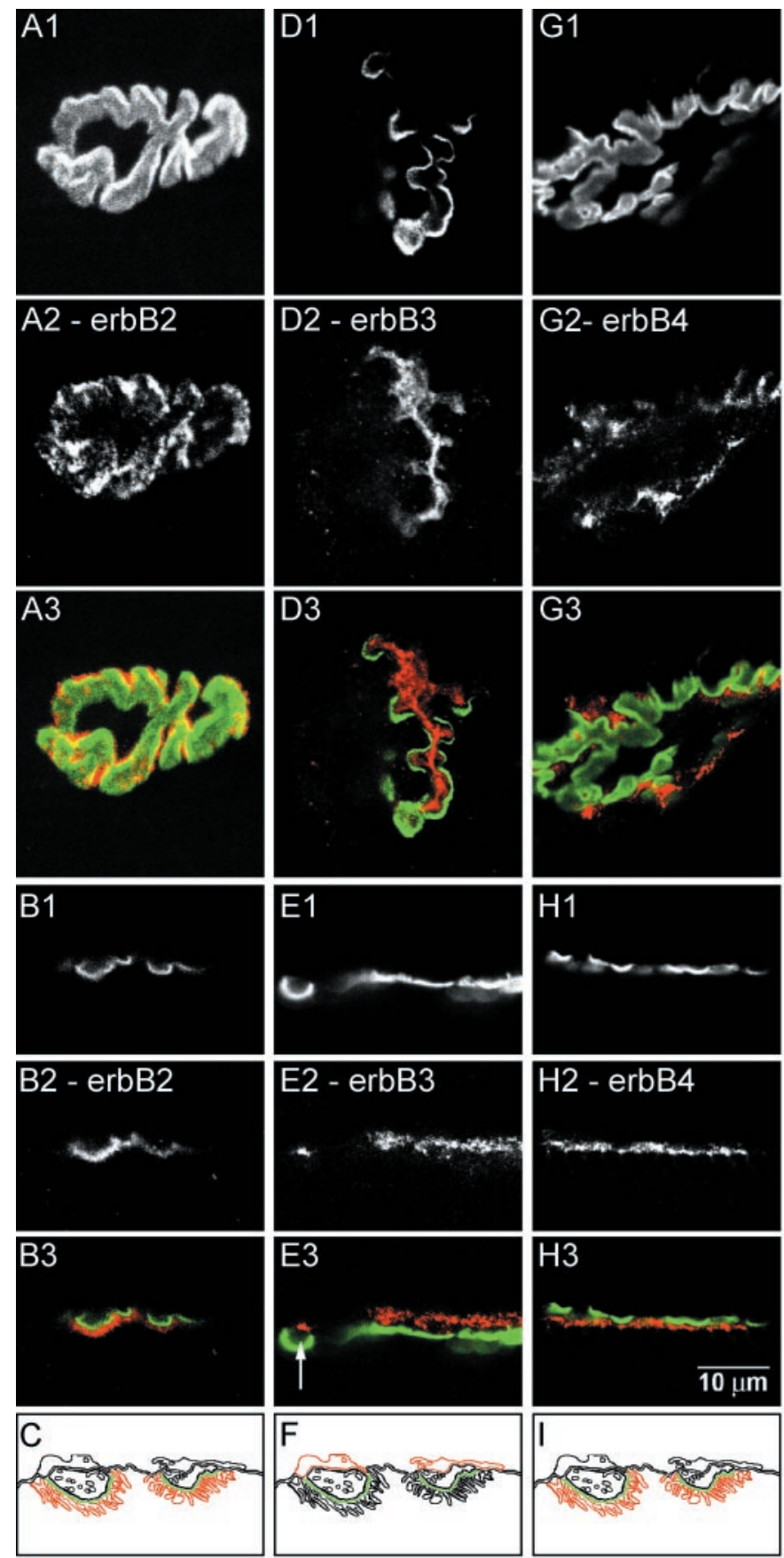

Figure 3. Confocal immunofluorescence analysis of erbB receptor distributions at adult rat gastrocnemius NMJ. En face $(A 1, D 1, G 1)$ and side view $(B 1, E 1, H 1)$ images of NMJs stained with Bodipy- $\alpha$ Btx. The NMJs were double-labeled with antibodies against erbB2 $(A 2, B 2)$, erbB3 $(D 2, E 2)$, and erbB4 $(G 2, H 2)$ and visualized using a Cy3-conjugated secondary antibody. Color overlays are shown with $\alpha$-Btx in green and the respective antibody in red $(A 3-H 3)$. Schematics of these distributions are shown in $C, F$, and $I$. ErbB2 is enriched in the secondary folds. ErbB3 is enriched in the Schwann cell. A gap between the primary gutter and the erbB3 immunoreactivity can be seen in E3 (arrow). ErbB4 is enriched in the secondary folds. Scale bar, $10 \mu \mathrm{m}$.

was indicative of a primary gutter localization. When examined in side view (Fig. $4 H$ ), immunoreactivity for MuSK overlapped well with Bodipy- $\alpha$ Btx, although the MuSK signal appears broader both above and below the Bodipy- $\alpha$ Btx. Nevertheless, the regions of most intense signal from the two images overlapped.

The distribution of agrin immunofluorescence at the NMJ was most consistent with agrin being concentrated at either the axon terminal or in the synaptic basal lamina, or both. Viewed en face 

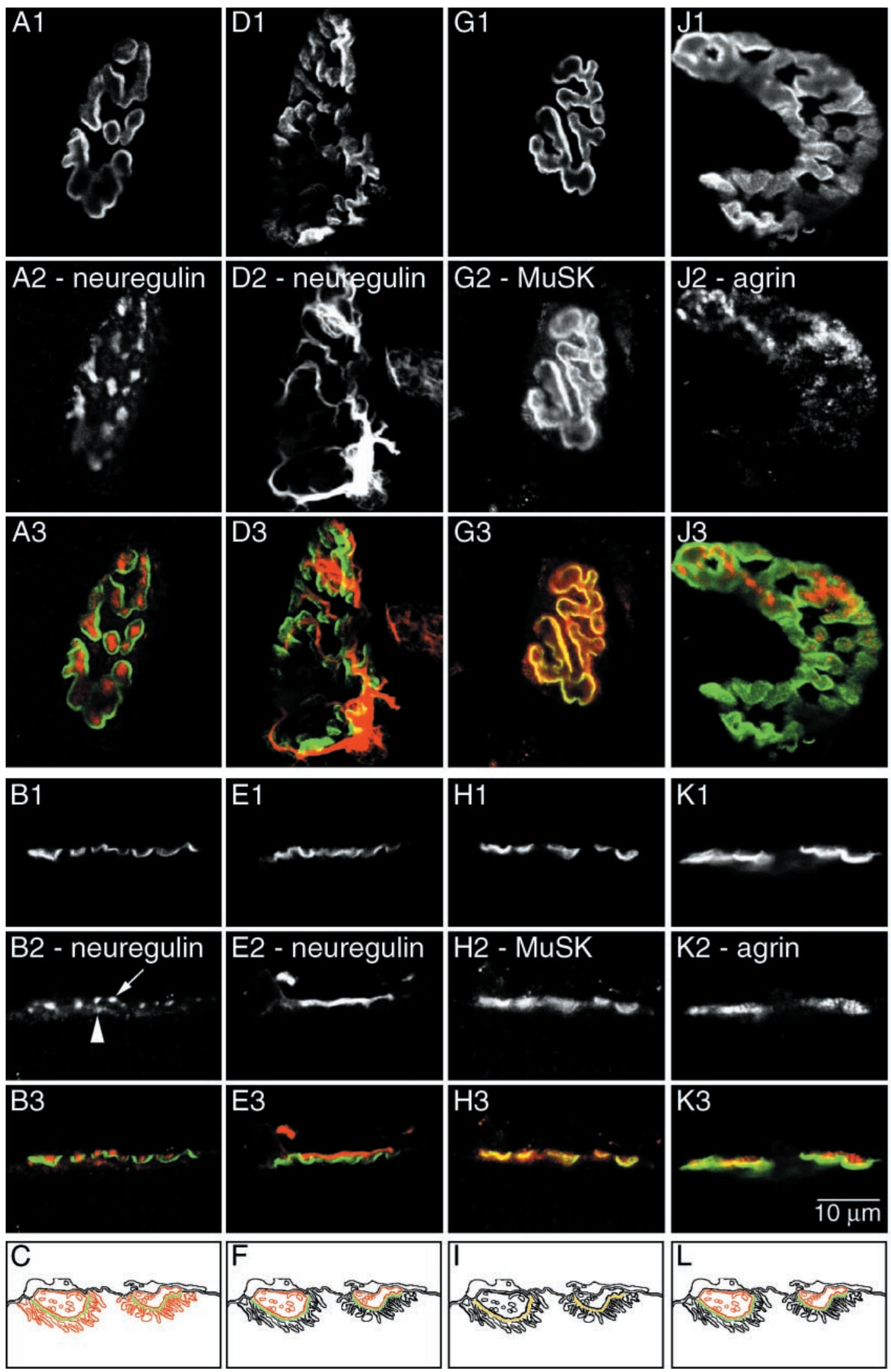

Figure 4. Confocal immunofluorescence analysis of neuregulin, MuSK, and agrin at adult rat gastrocnemius NMJ. En face (A1, D1, G1, J1) and side view $(B 1, E 1, H 1, K 1)$ images of NMJs stained with Bodipy- $\alpha$ Btx. The NMJs were double-labeled with antibodies against neuregulin-1914 (A2, B2), neuregulin-HM $24(D 2, E 2)$, MuSK $(G 2, H 2)$, and agrin $(J 2, K 2)$ and visualized using a Cy3-conjugated secondary antibody. Color overlays are shown with $\alpha$-Btx in green and the respective antibody in red (A3-K3). Schematics of these distributions are shown in $C, F, I$, and $L$. The neuregulin 1914 antibody recognizes neuregulin isoform(s) localized to the axon terminal (arrow) and the secondary folds (arrowhead). The neuregulin HM 24 antiserum recognizes neuregulin isoform(s) localized to the Schwann cell. MuSK is enriched in the primary gutter. Agrin appears enriched in the axon terminal and the basal lamina. Scale bar, $10 \mu \mathrm{m}$. 
(Fig. $4 J$ ), agrin immunoreactivity appeared to be localized to the primary gutter region. When examined in side view (Fig. $4 K$ ), agrin appeared offset on the presynaptic side of the primary gutter region with some overlap of the two signals. In Figure $4 J$, the agrin immunoreactivity does not appear to completely extend over the surface of the synapse. The immunofluorescence of the agrin antibody was not as robust as the other antibodies, which made it difficult to obtain crisp images of its distribution. However, our impression from viewing many NMJs is that agrin immunoreactivity is generally distributed over the entire synapse.

\section{DISCUSSION}

In this study, we used epifluorescence and confocal fluorescence microscopy to determine the relative distribution at the NMJ for the ligands and receptors of the neuregulin and agrin signaling pathways. Analysis of muscle cross sections at $20 \times$ magnification revealed that immunoreactivities for the erbB receptors, neuregulin, agrin, and MuSK were all clearly enriched at the NMJ. Analysis by confocal microscopy at $60 \times$ magnification $(1.40 \mathrm{NA})$ revealed that the neuregulin receptors erbB 2 and erbB4 were localized to the depths of the secondary folds, whereas erbB3 was associated with terminal Schwann cells. Neuregulin was found in the secondary folds, optimally positioned to interact with postsynaptic erbB2/4 receptors, whereas the GGF II isoform was associated with terminal Schwann cells, positioned to interact with erbB3 heterodimers. MuSK and agrin were both found in the primary gutter region, at a distance from postsynaptic erbB receptors.

\section{Localization of erbB3 to Schwann cells}

Our results show that erbB3 at the NMJ is localized to Schwann cells. Schwann cells are known to synthesize erbB3 (Vartanian et al., 1997). Myelinating Schwann cells along the nerve tract express erbB3 in adult rats and upregulate erbB3 expression after denervation (Carroll et al., 1997; Kwon et al., 1997). We found no evidence for either erbB2 or erbB4 on Schwann cells. Because erbB3 does not form functional homodimers, the manner in which terminal Schwann cells transduce the neuregulin signal is unknown. Myelinating Schwann cells express erbB2 (Kwon et al., 1997), so it is certainly possible that terminal Schwann cells expresses levels of erbB2 or erbB4 below our level of detection. Although erbB3-deficient mice die soon after birth, they are able to form NMJs. Such mutant animals lack both Schwann cells and Schwann cell precursors (Riethmacher et al., 1997).

We were unable to immunohistochemically detect erbB3 in myofibers. Moscoso et al. (1995) reported that erbB3 mRNA was expressed in muscle, although mRNA levels were not enriched in the synaptic region. There may be low levels of erbB3 protein present in the extra-junctional sarcolemma, but consistent with previous results (Altiok et al., 1995; Moskoso et al., 1995; Zhu et al., 1995), we did not detect any in muscle cross sections (Fig. 1). ErbB3 immunoreactivity remains at the NMJ after denervation (Moscoso et al., 1995). We believe that this immunoreactivity may be attributable to Schwann cells. Although Schwann cell processes retract from the primary gutter region after denervation, the Schwann cell bodies remain in the area of the synapse for several weeks (Trachtenberg and Thompson, 1997; Culican et al., 1998). It is possible, however, that muscle upregulates expression of erbB3 at the NMJ after denervation. In cultured myotubes, erbB3 is expressed and phosphorylated in response to neuregulin. At this stage in development, erbB3 may be involved in other roles, such as regulation of myotube fusion and myogenic differentiation (Kim et al., 1999). ErbB3 is absent from the NMJs of rapsyn-deficient mice (Gautam et al., 1995). On the basis of our results, a possible interpretation of this observation is that terminal Schwann cells only express erbB3 in the context of a fully mature NMJ.

\section{Localization of erbB2 and erbB4 in the secondary folds}

The erbB family of receptor tyrosine kinases signals through the formation of homodimers and heterodimers on ligand binding. Each specific dimer combination is capable of signaling a distinct biological response (Tzahar et al., 1994; Chen et al., 1996). Only erbB3 and erbB4 are capable of directly binding neuregulin, and erbB2 must form heterodimers with either erbB3 or erbB4 to respond to neuregulin. Although analysis of relative levels of erbB expression in muscle was not performed in this study, previous studies indicate that mRNA levels of erbB2 and erbB3 are greater than erbB4 in whole muscle (Zhu et al., 1995).

The postsynaptic enrichment of erbB2 and erbB4 that we observed suggests that the most likely functional neuregulin receptor in muscle at the NMJ is a heterodimer between erbB2 and erbB4, although homodimers of erbB4 could potentially exist. ErbB2deficient mice have been generated. These mice still show synaptic enrichment of AChR mRNA (Woldeyesus et al., 1999; Lin et al., 2000). This suggests that ErbB2 is not essential to regulate synaptic transcription. If this is the case, then it is likely that homodimers of erbB4 are able to initiate the neuregulin signaling cascade. ErbB receptors located in the depths of the secondary folds are in close physical proximity to subsynaptic nuclei. This arrangement may help promote the ability of neuregulin to signal to the nucleus.

Because erbB2 and erbB4 are localized to the secondary folds, they cannot be enriched at the NMJ because of direct interactions with rapsyn. Rapsyn plays an essential role in aggregating AChRs in the primary gutter, but its distribution does not extend to the depths of the secondary folds (Flucher and Daniels, 1989), and it therefore cannot interact directly with erbB2 and erbB4.

ErbB2 and erbB4 might be localized to the secondary folds via interactions with a structural protein(s). Sodium channels are known to interact with spectrin, ankyrinG, and syntrophin, proteins that may play a structural role in organizing the secondary folds (Gee et al., 1998; Wood and Slater, 1998). Recent evidence suggests a role for proteins containing the PDZ protein interaction domain in localization of erbB2 and erbB4, but interestingly not erbB3. The $\mathrm{C}$ terminus of erbB4 contains the canonical sequence for interaction with PDZ domains: S/T, X, V. Syntrophin and spectrin contain PDZ domains and are enriched at the NMJ (Garcia et al., 2000). Furthermore, the $\beta 2$ isoform of syntrophin occurs mainly in the secondary folds (Kramarcy and Sealock, 2000).

\section{Neuregulin is present in the secondary folds and associated with terminal Schwann cells}

At least 14 splice variants of neuregulin-1 are known to exist (Peles et al., 1993), although the expression of each isoform at the NMJ has not been determined. Here we show that antibodies against two neuregulin isoforms detect neuregulin differentially positioned to interact with erbB receptors on the Schwann cell and in the secondary folds. GGF II, the neuregulin splice variant containing the $\beta 3$ exon, has been implicated in signaling to glia or Schwann cells (Florini et al., 1996). Consistent with this observation, we have now shown that an antiserum against the $\beta 3$ peptide was associated with terminal Schwann cells located at the NMJ.

The second neuregulin antibody was generated against the Ig and epidermal growth factor (EGF) common domains and included the $\alpha 2$ insert (Sandrock et al., 1995). This antibody detects neuregulin in the secondary folds and neuregulin associated with the axon terminal. This antibody does not appear to recognize neuregulin associated with the Schwann cell, although $\beta 3$ isoforms of neuregulin are thought to contain the EGF common domain. Although the epitopes that are recognized by this antibody have not been defined, it would stand to reason that the EGF common domain was not a very antigenic region of the protein. Although levels of neuregulin in the secondary folds appear relatively low, they are correctly positioned to interact with erbB2 and erbB4 on the muscle sarcolemma.

The axon terminal, Schwann cell, and muscle fiber are possible sources of neuregulin. Our results do not address the origin of neuregulin localized at the NMJ. Many isoforms of neuregulin are originally synthesized containing a transmembrane as well as a cytosolic region. These proteins are thought to be proteolytically processed to yield the mature extracellular signaling protein. Neu- 
regulin released into the extracellular matrix interacts with components of the basal lamina (Loeb et al., 1999). Neuregulin is also a heparin binding protein, and one manner by which it might become localized to the secondary folds or Schwann cell is through binding interactions with components of the basal lamina.

\section{MuSK and agrin colocalize in the primary gutter}

The majority of MuSK immunoreactivity colocalizes with Bodipy$\alpha$ Btx fluorescence in the primary gutter. MuSK was therefore well positioned to initiate a signal transduction cascade resulting in the recruitment of rapsyn and AChR to the primary gutter. We detected low levels of MuSK both above and below the Bodipy- $\alpha$ Btx fluorescence. The fact that some of the lower signal exists presynaptically argues that this may be because of a background signal.

Using a pan-agrin antibody on muscle cross sections, we detected agrin only at the NMJ and not extrasynaptically in the muscle fiber. This is consistent with restriction of agrin to the NMJ postnatally (Fallon and Gelfman, 1989; Bowe and Fallon, 1995). Closer inspection using confocal microscopy revealed agrin localized at the axon terminal and in the synaptic cleft of the primary gutter. There it was positioned to interact with MuSK in the primary gutter.

\section{Differential localization of the signaling pathways}

The involvement of agrin and MuSK in the localization of the primary gutter proteins rapsyn and AChR has been well studied (Ferns et al., 1992; Glass et al., 1996; Fuhrer et al., 1997; Burgess et al., 1999). We have shown that MuSK is localized to the primary gutter where it can interact with agrin. ErbB2 and erbB4 are enriched in the secondary folds of the postsynaptic membrane where they can interact with neuregulin. Localization of MuSK and agrin essentially to the primary gutter implies that erbB receptors and other secondary fold proteins are not enriched at the NMJ because of direct protein-protein interactions with MuSK. The restricted distributions of neuregulin and agrin argue against free diff usion of these ligands in the synaptic cleft and suggest that their localization is regulated by interactions with other components of the basal lamina.

\section{REFERENCES}

Altiok N, Bessereau J-L, Changeux J-P (1995) ErbB3 and ErbB2/neu mediate the effect of heregulin on acetylcholine receptor gene expression in muscle: differential expression at the endplate. EMBO J 14:4258-4266.

Altiok N, Altiok S, Changeux J-P (1997) Heregulin-stimulated acetylcholine receptor gene expression in muscle: requirement for MAP kinase and evidence for a parallel inhibitory pathway independent of electrical activity. EMBO J 16:717-725.

Apel ED, Glass DJ, Moscoso LM, Yancopoulos GD, Sanes JR (1997) Rapsyn is required for MuSK signaling and recruits synaptic components to a MuSK-containing scaffold. Neuron 18:623-635.

Bewick GS, Nicholson LVB, Young C, O'Donnell E, Slater CR (1992) Different distributions of dystrophin and related proteins at nerve-muscle junctions. NeuroReport 3:857-860.

Bewick GS, Young C, Slater CR (1996) Spatial relationships of utrophin, dystrophin, beta-dystroglycan and beta-spectrin to acetylcholine receptor clusters during postnatal maturation of the rat neuromuscular junction. J Neurocytol 25:367-379.

Bowe MA, Fallon JR (1995) The role of agrin in synapse formation. Annu Rev Neurosci 18:443-462.

Buckley K, Kelly RB (1985) Identification of a transmembrane glycoprotein specific for secretory vesicles of neural and endocrine cells. J Cell Biol 100:1284-1294.

Burgess RW, Nguyen QT, Son YJ, Lichtman JW, Sanes JR (1999) Alternatively spliced isoforms of nerve- and muscle-derived agrin: their roles at the neuromuscular junction. Neuron 23:33-44.

Carroll SL, Miller ML, Frohnert PW, Kim SS, Corbett JA (1997) Expression of neuregulins and their putative receptors, ErbB2 and ErbB3, is induced during Wallerian degeneration. J Neurosci 17:1642-1659.

Chen X, Levkowitz G, Tzahar E, Karunagaran D, Lavi S, Ben-Baruch N, Leitner O, Ratzkin BJ, Bacus SS, Yarden Y (1996) An immunological approach reveals biological differences between the two NDF/heregulin receptors, ErbB-3 and ErbB-4. J Biol Chem 271:7620-7629.

Chu GC, Moscoso LM, Sliwkowski MX, Merlie JP (1995) Regulation of the acetylcholine receptor $\epsilon$ subunit gene by recombinant ARIA: an in vitro model for transynaptic gene regulation. Neuron 14:329-339.

Cohen I, Rimer M, Lomo T, McMahan UJ (1997) Agrin-induced postsynaptic-like apparatus in skeletal muscle fibers in vivo. Mol Cell Neurosci 9:237-253.
Culican SM, Nelson CC, Lichtman JW (1998) Axon withdrawal during synapse elimination at the neuromuscular junction is accompanied by disassembly of the postsynaptic specialization and withdrawal of Schwann cell processes. J Neurosci 18:4953-4965.

Dechiara TM, Bowen DC, Valenzuela DM, Simmons MV, Poueymirou WT, Thomas S, Kinetz E, Compton DL, Rojas E, Park JS, Smith C, Distefano PS, Glass DJ, Burden SJ, Yancopoulos GD (1996) The receptor tyrosine kinase $\mathrm{MuSK}$ is required for neuromuscular junction formation in vivo. Cell 85:501-512.

Fallon JR, Gelfman CE (1989) Agrin-related molecules are concentrated at acetylcholine receptor clusters in normal and aneural developing muscle. J Cell Biol 108:1527-1535.

Ferns M, Hoch W, Campanelli JT, Rupp F, Hall ZW, Scheller RH (1992) RNA splicing regulates agrin-mediated acetylcholine receptor clustering activity on cultured myotubes. Neuron 8:1079-1086.

Fischbach GD, Rosen KM (1997) ARIA: a neuromuscular junction neuregulin. Annu Rev Neurosci 20:429-458.

Florini JR, Samuel DS, Ewton DZ, Kirk C, Sklar RM (1996) Stimulation of myogenic differentiation by a neuregulin, glial growth factor 2 . J Biol Chem 271:12699-12702.

Flucher BE, Daniels MP (1989) Distribution of $\mathrm{Na}+$ channels and ankyrin in neuromuscular junctions is complementary to that of acetylcholine receptors and the $43 \mathrm{kD}$ protein. Neuron 3:163-175.

Froehner SC, Luetje CW, Scotland PB, Patrick J (1990) The postsynaptic $43 \mathrm{~K}$ protein clusters muscle nicotinic acetylcholine receptors in Xenopus oocytes. Neuron 5:403-410.

Fromm L, Burden SJ (1998) Synapse-specific and neuregulin-induced transcription require an Ets site that binds GABPalpha/GABPbeta. Genes Dev 12:3074-3083.

Fuhrer C, Sugiyama JE, Taylor RG, Hall ZW (1997) Association of muscle-specific kinase MuSK with the acetylcholine receptor in mammalian muscle. EMBO J 16:4951-4960.

Garcia RAG, Vasudevan K, Buonanno A (2000) The neuregulin receptor ErbB-4 interacts with PDZ-containing proteins at neuronal synapses. Proc Natl Acad Sci USA 97:3596-3601.

Gautam M, Noakes PG, Mudd J, Nichol M, Chu GC, Sanes JR, Merlie JP (1995) Failure of postsynaptic specialization to develop at neuromuscular junctions of rapsyn-deficient mice. Nature 377:232-236.

Gautam M, Noakes PG, Moscoso L, Rupp F, Scheller RH, Merlie JP, Sanes JR (1996) Defective neuromuscular synaptogenesis in agrin-deficient mutant mice. Cell 85:525-535.

Gee SH, Madhavan R, Levinson SR, Caldwell JH, Sealock R, Froehner SC (1998) Interaction of muscle and brain sodium channels with multiple members of the syntrophin family of dystrophin-associated proteins. J Neurosci 18:128-137.

Glass DJ, Yancopoulos GD (1997) Sequential roles of agrin, MuSK and rapsyn during neuromuscular junction formation. Curr Opin Neurobiol 7:379-384.

Glass DJ, Bowen DC, Stitt TN, Radziejewski C, Bruno J, Ryan TE, Gies DR, Shah S, Mattsson K, Burden SJ, Distefano PS, Valenzuela DM, Dechiara TM, Yancopoulos GD (1996) Agrin acts via a MuSK receptor complex. Cell 85:513-523.

Goodearl ADJ, Yee AG, Sandrock Jr AW, Corfas G, Fischbach GD (1995) ARIA is concentrated in the synaptic basal lamina of the developing chick neuromuscular junction. J Cell Biol 130:1423-1434.

Guy PM, Platko JV, Cantley LC, Cerione RA, Carraway IKL (1994) Insect cell-expressed p180 erbB3 possesses an impaired tyrosine kinase activity. Proc Natl Acad Sci USA 91:8132-8136.

Hoch W, Ferns M, Campanelli JT, Hall ZW, Scheller RH (1993) Developmental regulation of highly active alternatively spliced forms of agrin. Neuron 11:479-490.

Jones G, Moore C, Hashemolhosseini S, Brenner HR (1999) Constitutively active MuSK is clustered in the absence of agrin and induces ectopic postsynaptic-like membranes in skeletal muscle fibers. J Neurosci 19:3376-3383

Kim DG, Chi SS, Lee KH, Rhee SY, Kwon YH, Chung CH, Kwon HM, Kang MS (1999) Neuregulin stimulates myogenic differentiation in an autocrine manner. J Biol Chem 274:15395-15400.

Kramarcy NR, Sealock R (2000) Syntrophin isoforms at the neuromuscular junction: developmental time course and differential localization. Mol Cell Neurosci 15:262-274.

Kwon YK, Bhattacharyya A, Alberta JA, Giannobile WV, Cheon K, Stiles CD, Pomeroy SL (1997) Activation of ErbB2 during Wallerian degeneration of sciatic nerve. J Neurosci 17:8293-8299.

Lenferink AEG, Pinkas-Kramarski R, van de Poll MLM, van Vugt MJH, Klapper LN, Tzahar E, Waterman H, Sela M, van Zeolen EJJ, Yarden Y (1998) Differential endocytic routing of homo- and hetero-dimeric ErbB tyrosine kinases confers signaling superiority to receptor heterodimers. EMBO J 17:3385-3397.

Lin WC, Sanchez HB, Deerinck T, Morris JK, Ellisman M, Lee KF (2000) Aberrant development of motor axons and neuromuscular synapses in erbB2-deficient mice. Proc Natl Acad Sci USA 97:1299-1304.

Loeb JA, Khurana TS, Robbins JT, Yee AG, Fischbach GD (1999) Expression patterns of transmembrane and released forms of neuregulin during spinal cord and neuromuscular synapse development. Development 126:781-791. 
Marchionni MA, Goodearl ADJ, Chen MS, Bermingham-McDonogh O, Kirk C, Hendricks M, Danehy F, Misumi D, Sudhalter J, Kobayashi K, Wroblewski D, Lynch C, Baldassare M, Hiles I, Davis JB, Hsuan JJ, Totty NF, Otsu M, McBurney RN, Waterfield MD, Stroobant P, Gwynne D (1993) Glial growth factors are alternatively spliced erbB2 ligands expressed in the nervous system. Nature 362:312-318.

Martinou J-C, Falls DL, Fischbach GD, Merlie JP (1991) Acetylcholine receptor-inducing activity stimulates expression of the $\epsilon$-subunit gene of the muscle acetylcholine receptor. Proc Natl Acad Sci USA 88:7669-7673.

Meier T, Masciulli F, Moore C, Schoumacher F, Eppenberger U, Denzer AJ, Jones G, Brenner HR (1998) Agrin can mediate acetylcholine receptor gene expression in muscle by aggregation of muscle-derived neuregulins. J Cell Biol 141:715-726.

Moscoso LM, Chu GC, Gautam M, Noakes PG, Merlie JP, Sanes JR (1995) Synapse-associated expression of an acetylcholine receptorinducing protein, ARIA/heregulin, and its putative receptors, ErbB2 and ErbB3, in developing mammalian muscle. Dev Biol 172:158-169.

Peles E, Ben-Levy R, Tzahar E, Liu N, Wen D, Yarden Y (1993) Celltype specific interaction of Neu differentiation factor ( $\mathrm{NDF} /$ heregulin) with Neu/HER-2 suggests complex ligand-receptor relationships. EMBO J 12:961-971.

Phillips W, Kopta C, Blount P, Gardner P, Steinbach J-H, Merlie J (1991) ACh receptor-rich membrane domains organized in fibroblasts by recombinant 43-kilodalton protein. Science 251:568-570.

Pinkas-Kramarski R, Shelly M, Glathe S, Ratzkin BJ, Yarden Y (1996) Neu differentiation factor/neuregulin isoforms activate distinct receptor combinations. J Biol Chem 271:19029-19032.

Ramarao MK, Cohen JB (1998) Mechanism of nicotinic acetylcholine receptor cluster formation by rapsyn. Proc Natl Acad Sci USA 95:4007-4012.

Riethmacher D, Sonnenberg-Riethmacher E, Krinkmann V, Yamaai T, Lewin GR, Birchmeier C (1997) Severe neuropathies in mice with targeted mutations in the ErbB3 receptor. Nature 389:725-730.

Sandrock Jr AW, Goodearl ADJ, Yin Q-W, Chang D, Fischbach GD
(1995) ARIA is concentrated in nerve terminals at neuromuscular junctions and at other synapses. J Neurosci 15:6124-6136.

Sanes JR, Lichtman JW (1999) Development of the vertebrate neuromuscular junction. Annu Rev Neurosci 22:389-442.

Sapru MK, Florance SK, Kirk C, Goldman D (1998) Identification of a neuregulin and protein-tyrosine phosphatase response element in the nicotinic acetylcholine receptor $\epsilon$ subunit gene: regulatory role of an Ets transcription factor. Proc Natl Acad Sci USA 95:1289-1294.

Si J, Wang Q, Mei L (1999) Essential roles of c-JUN and c-JUN $\mathrm{N}$-terminal kinase (JNK) in neuregulin-increased expression of the acetylcholine receptor $\epsilon$-subunit. J Neurosci 19:8498-8508.

Trachtenberg JT, Thompson WJ (1997) Nerve terminal withdrawal from rat neuromuscular junctions induced by neuregulin and Schwann cells. J Neurosci 17:6243-6255.

Tzahar E, Levkowitz G, Karunagaran D, Yi L, Peles E, Lavi S, Chang D, Liu N, Yayon A, Wen D, Yarden Y (1994) ErbB-3 and ErbV-4 function as the respective low and high affinity receptors of all Neu differentiation factor/heregulin isoforms. J Biol Chem 269:25226-25233.

Vartanian T, Goodearl A, Viehover A, Fischbach G (1997) Axonal neuregulin signals cells of the oligodendrocyte lineage through activation of HER4 and schwann cells through HER2 and HER3. J Cell Biol 137:211-220.

Waterman H, Sabanai I, Geiger B, Yarden Y (1998) Alternative intracellular routing of ErbB receptors may determine signaling potency. J Biol Chem 273:13819-13827.

Woldeyesus MT, Britsch S, Riethmacher D, Xu L, SonnenbergRiethmacher E, Abou-Rebyeh F, Harvey R, Caroni P, Birchmeier C (1999) Peripheral nervous system defects in erbB2 mutants following genetic rescue of heart development. Genes Dev 13:2538-2548.

Wood SJ, Slater CR (1998) beta-spectrin is colocalized with both voltagegated sodium channels and ankyrin $(\mathrm{G})$ at the adult rat neuromuscular junction. J Cell Biol 140:675-684.

Zhu X, Lai C, Thomas S, Burden SJ (1995) Neuregulin receptors, erbB3 and erbB4, are localized at neuromuscular synapses. EMBO J 14:58425848 . 